\title{
Intervertebral Disc Degeneration
}

\section{The Role of the Mitochondrial Pathway in Annulus Fibrosus Cell Apoptosis Induced by Overload}

François Rannou, ${ }^{* \dagger}$ Tzong-Shyuan Lee, ${ }^{*}$ Rui-Hai Zhou, ${ }^{*}$ Jennie Chin, ${ }^{\ddagger}$ Jeffrey C. Lotz, ${ }^{\ddagger}$ Marie-Anne Mayoux-Benhamou, ${ }^{\dagger}$ Jacques Patrick Barbet, ${ }^{\S}$ Alain Chevrot, ${ }^{\pi}$ and John Y.-J. Shyy*

From the Division of Biomedical Sciences," University of California, Riverside, California; Orthopedic Bioengineering Laboratory, ${ }^{\ddagger}$ Department of Orthopedic Surgery, University of California, San Francisco, California; Rehabilitation and Spine Center, ${ }^{\dagger}$ Division of Histology-Embryology-Cytogenetics, ${ }^{\S}$ and Division of Radiology, "T Cochin Hospital, University of Paris V, Paris, France

Degeneration of the intervertebral disk (IVD) is a major pathological process implicated in low back pain and is a prerequisite to disk herniation. Although mechanical stress is an important modulator of the degeneration, the underlying molecular mechanism remains unclear. The association of human IVD degeneration, assessed by magnetic resonance imaging, with annulus fibrosus cell apoptosis and anti-cytochrome $c$ staining revealed that the activation of the mitochondria-dependent apoptosome was a major event in the degeneration process. Mouse models of IVD degeneration were used to investigate the role of the mechanical stress in this process. The application of mechanical overload (1.3 MPa) for 24 hours induced annulus fibrosus cell apoptosis and led to severe degeneration of the mouse disks. Immunostaining revealed cytochrome $c$ release but not Fas-L generation. The role of the caspase-9-dependent mitochondrial pathway in annulus fibrosus cell apoptosis induced by overload was investigated further with the use of cultured rabbit IVD cells in a stretch device. Mechanical overload (15\% area change) induced apoptosis with increased caspase- 9 activity and decreased mitochondrial membrane potential. Furthermore, $Z$ LEHD-FMK, a caspase-9 inhibitor, but not Z-IETDFMK, a caspase-8 inhibitor, attenuated the overloadinduced apoptosis. Our results from human samples, mouse models, and annulus fibrosus culture experiments demonstrate that the mechanical overload-induced IVD degeneration is mediated through the mi- tochondrial apoptotic pathway in IVD cells. ( $A m$ J Pathol 2004, 164:915-924)

The causes of low back pain have been suggested to involve genetic, environmental, and psychosocial factors. ${ }^{1-5}$ Recent studies suggest that the main anatomical structure implicated in low back pain is the intervertebral disk (IVD) and the pathogenic process is its degeneration, which predisposes disk herniation. ${ }^{6-10}$ To date, the pathophysiologic causes of IVD degeneration at the molecular level are still unclear. The IVD is composed of two distinct but interdependent tissues: a gelatinous center, known as the nucleus pulposus (NP), and several surrounding coaxial lamellae that form the inner and outer annulus fibrosus (AF). This unique structural feature allows the IVD to constrain motion at high loads and provide flexibility at low loads. When degeneration occurs, it is difficult to distinguish the two anatomical parts of the AF. Experimental animal models and clinical evidence suggest that excessive mechanical loading enhances the disk degeneration, which indicates that IVD cells can sense and convert applied overloads to biological signals that result in tissue responses. ${ }^{11-14}$

Constituting $1 \%$ volume of the disk, IVD cells play an important role in maintaining the integrity of the disk by producing proteoglycans, type I and type II collagen, and factors involved in extracellular-matrix (ECM) turnover, including metalloproteinases, prostaglandins, and nitric oxide..$^{15-19}$ In the healthy IVD, the rates of synthesis and breakdown of the ECM are in equilibrium because of the intricate regulation by growth factors and cytokines. In the disease state, IVD degeneration has been suggested to be associated with a loss of tissue cellularity through apoptotic-related processes that lead to a diminished generation, organization, and repair of the ECM. ${ }^{12,20,21}$ Apparently, the homeostasis of the ECM in the IVD is

Supported in part by funds from Société Française de Rhumatologie Bourse du Ministère des Affaires Etrangères du Programme Lavoisier. Fondation Bettencourt Schueller, Fondation Philippe, Fondation des Treilles, National Institutes of Health (NIH) grant HL60789 (to J.S.), and NIH grant AR46173 (to J.C.L.).

Accepted for publication November 18, 2003.

Address reprint requests to John Y-J. Shyy, Ph.D., Division of Biomedical Sciences, University of California, Riverside, Riverside, CA 925210121. E-mail: john.shyy@ucr.edu. 
regulated by mechanical loads as well. ${ }^{22-25}$ In some circumstances, mechanical overload impairs ECM homeostasis, causing the onset of IVD degeneration, ${ }^{22-24}$ which is evidenced by the positive correlation between spinal loading and IVD degeneration. ${ }^{14}$ Thus, results from these previous studies suggest the pivotal role of mechanical stress in inducing IVD cell apoptosis and ECM degradation, which may lead to IVD degeneration.

Apoptosis plays a central role in the homeostasis of all organisms in normal development and tissue turnover. ${ }^{26}$ The activation of initiator caspases is the central event in the effector phase of apoptosis. ${ }^{27}$ Two processes implicating protein-protein interaction lie immediately upstream of the apoptotic initiators caspase- 8 and -9 . The first process or extrinsic pathway can be induced by the ligands of the tumor necrosis factor (TNF)-receptor superfamily. These receptors can activate caspase- 8 via the Fas-associated death domain protein, which is able to bridge members of the TNF-receptor superfamily to the caspase-8 proenzyme. ${ }^{28}$ In turn, caspase-8 directly cleaves and activates the effector caspase-3. The second or intrinsic pathway involving caspase-9 as the initiator originates from mitochondria. Stressed mitochondria release a set of molecules, including cytochrome $c$ and Apaf-1, to form the apoptosome molecular cluster that activates caspase- $9^{29}$ and its effector caspase-3. In addition, this pathway could be indirectly activated by caspase-8 through the cleavage of Bid (a pro-death $\mathrm{Bcl}-2$ family protein). ${ }^{30}$

We report here a mitochondria-dependent IVD cell apoptosis that occurs in human IVD degeneration and in mouse models with IVD degeneration caused by mechanical overload. The hypothesis that caspase- 9 mediates the mechanical-overload-induced apoptosis was further tested by in vitro experiments involving a stretch device with use of cultured rabbit IVD cells.

\section{Materials and Methods}

\section{Human IVD Samples}

Lumbar spines were removed from 11 cadavers of patients of varying ages with no history of spinal trauma or inflammatory disease. To avoid dehydration of the disks, spines with extensive adjacent tissues were removed within 48 hours following death, in full agreement with French legislation on ethical rules. The removed spines immediately underwent magnetic resonance imaging (MRI) (1.5-T; TR/TE: 4000/95 ms for sagittal images and $640 / 14$ ms for axial images). With agreement from two rheumatologists and one radiologist, IVD degeneration was determined by MRI signal intensities of L3-L4, L4-L5, and L5-S1 disks. The following grading system for T2weighted signal intensity was used to assess degeneration: "no degeneration," indicated by the enhanced signal intensity in the center (grade 0); "moderate degeneration," indicated by inhomogeneous and decreased signal intensity (grade 1); and "severe degeneration," indicated by decreased overall signal intensity (grade 2). ${ }^{31}$ Immediately after MRI, all adjacent tissues were removed from the spines, and the specimens were then fixed, decalcified, and embedded in paraffin. Midsagittal serial sections $(6-8 \mu \mathrm{m})$ were obtained for immunostaining. All specimens were kept at $4^{\circ} \mathrm{C}$ between various procedures.

\section{Mouse Model for IVD Degeneration}

An in vivo mouse model of IVD degeneration was used to investigate the mechanism by which mechanical overload induces disk degeneration. ${ }^{12}$ The animal protocol was approved by the University of California, San Francisco Committee on Animal Research. Briefly, in group one $(n=4)$, mouse tail disks were loaded with an external compression device in vivo as previously described. ${ }^{12}$ Compressive stress was applied at one magnitude (1.3 $\mathrm{MPa}$ ) between the ninth and 10th caudal vertebrae for 24 hours before the mice were sacrificed. This magnitude reflects human spinal overload $(1500 \mathrm{~N}) .{ }^{12}$ In group two $(n=3)$, the "sham" group, the compression device was applied to mouse tails for 24 hours without stress. Mice in group three $(n=3)$, undergoing no surgery, were used for additional controls. Immediately after the mice were sacrificed, spines were dissected and the subject disks harvested, fixed, and embedded in paraffin. Midsagittal serial sections $(5 \mu \mathrm{m})$ were obtained accordingly.

\section{Apoptosis and Immunohistochemistry Studies for IVD Specimens}

The cells in the human and mouse paraffin serial sections of the IVD were counted manually after hematoxylin and eosin staining. The DNA fragmentation was assessed by use of the terminal deoxynucleotidyl transferase-mediated dUTP nick end-labeling (TUNEL) method (Boehringer Mannheim, Mannheim, Germany) as previously described. $^{32}$ Condensed or fragmented nuclei were defined as apoptotic cells. The nuclei were counterstained by DAPI to detect non-apoptotic cells and examined under a fluorescence microscope. For each IVD, cells in three different views from one section of AF and/or NP were counted by two of the co-authors using a microscope at $\times 100$ magnification after hematoxylin and eosin staining. The two investigators' average counts of the three views were pooled and subjected to statistical analysis. Results are presented as number of cells per square millimeter. The percentage of apoptotic cells was determined from random views of at least 400 cells. Results are presented as percentage of cells relative to the total number of cells. Sections were also incubated with rabbit anti-cytochrome $C$ antibody (1:50 dilution) (Santa Cruz Biotechnology, Santa Cruz, CA) or goat anti-Fas-L antibody (1:20 dilution) (Santa Cruz Biotechnology), followed by horseradish peroxidase-conjugated secondary antibodies. Sections were counterstained with hematoxylin. For immunohistochemical controls, sections were incubated with PBS without the primary antibodies. The specimens were viewed under a Nikon TE300 microscope. Results are presented as percentage of cells with or 
without cytochrome $c$ release relative to the total number of cells.

\section{Cell Culture and Cyclic Stretch Experiments}

Rabbit AF cells were isolated and cultured as previously described. ${ }^{33}$ The animal protocol was approved by the Committee on Animal Care of the University of California, Riverside. The isolated cells were maintained in Ham's F12 medium containing 10\% fetal bovine serum, 100 $\mathrm{IU} / \mathrm{ml}$ penicillin, and $100 \mu \mathrm{g} / \mathrm{ml}$ streptomycin (Gibco, Grand Island, NY). Only first-passage AF cells were used in this study, and the isolated cells maintained their phenotype until their use in stretch experiments. ${ }^{33}$ A stretch apparatus ${ }^{34}$ was used to apply uniform, sinusoidal cyclic stretch to confluent AF cells cultured on silicon elastic membranes (Specialty Manufacturing, Saginaw, MI) coated with collagen type I $\left(10 \mu \mathrm{g} / \mathrm{cm}^{2}\right.$; Sigma, St. Louis, $\mathrm{MO})$. The amplitude of the cyclic stretch was $15 \%$ area change, the frequency $0.1 \mathrm{~Hz}$, and the duration 24 hours. The static controls were cells cultured on elastic membranes never exposed to cyclic stretch. The positive controls for apoptosis were AF cells cultured on elastic membranes treated with staurosporine (Calbiochem, La Jolla, CA; $1 \mu \mathrm{mol} / \mathrm{L}$ in Ham's F12) for 10 hours. In some of the experiments, cell-permeable caspase-8 inhibitor ZIle-Glu-Thr-Asp (IETD)-FMK or caspase-9 inhibitor Z-LeuGlu-His-Asp (LEHD)-FMK (Calbiochem) was added to the culture media (100 $\mu \mathrm{mol} / \mathrm{L}) 2$ hours before the stretch experiments. This concentration of caspase inhibitors has been shown to inhibit caspase-8 and -9 specifically. ${ }^{35,36}$

\section{Apoptosis Analyses for in Vitro Stretch Experiments}

Ligation-mediated polymerase chain reaction (LM-PCR), Hoechst staining, and flow cytometric analyses were used to analyze the stretch-induced apoptosis. For LMPCR, the genomic DNA was isolated by use of the Puregene kit (Gentra Systems, Minneapolis, MN), and DNA fragmentation was investigated by use of a LM-PCR ladder assay kit (Clontech, Palo Alto, CA). For nuclear chromatin detection, AF cells were fixed in $4 \%$ paraformaldehyde and stained with $1 \mu \mathrm{g} / \mathrm{ml}$ Hoechst 33258 (Molecular Probes, Eugene, OR). Apoptotic cells were identified by the condensation and fragmentation of nuclei, which was examined under a Nikon TE300 microscope. For flow cytometric analysis, AF cells were detached from the elastic membranes, fixed with $70 \%$ ethanol, and suspended in PBS with $0.5 \mathrm{mg} / \mathrm{ml}$ RNase A (Sigma). After 30 minutes incubation, $50 \mu \mathrm{g} / \mathrm{ml}$ propidium iodide (PI) (Sigma) was added. Analyses were performed by counting $10^{4}$ cells with a fluorescence-activated cell sorter (FACS). The fraction of cells in the hypodiploid population was calculated from fluorescence frequency histograms and expressed as a proportion of total cells (\% of cell death).

\section{Measurement of Caspase-8 and -9 Activity and Mitochondrial Membrane Potential $(\Delta \Psi)$}

Enzymatic activity of caspase-8 and -9 was determined by colorimetric assay, which measured the release of the chromophore $p$-nitroaniline ( $p-\mathrm{NA}$ ) combined with a peptide substrate specific to caspase-8 (IETD-p-NA) or one specific to caspase-9 (LEHD-p-NA). Briefly, AF cells were lysed, and aliquots of $200 \mu \mathrm{g}$ of cell extracts were incubated at $37^{\circ} \mathrm{C}$ for 2 hours in the presence of the specific substrate in 96-well plates. Peptide cleavage was quantified by a colorimetric plate reader set at $405 \mathrm{~nm}$.

AF cells were stained with DiOC6, and flow cytometry was performed to examine $\Delta \Psi$. Non-fixed cells were stained with $40 \mathrm{nmol} / \mathrm{L}$ DiOC 6 at $37^{\circ} \mathrm{C}$ for 20 minutes. An amount of $10^{4}$ cells from each sample was counted with use of the FACS. The fraction of cells with a decrease in DiOC6 fluorescence was evaluated and expressed as a proportion of total cells.

\section{Statistics}

All experiments were performed independently at least three times. Student's $t$-test and analysis of variance were used to determine statistical significance.

\section{Results}

Involvement of the Mitochondria-

Dependent Apoptosome (MDA) in Human IVD Degeneration

According to the MRI signal intensity, 13, 14, and 6 disks from 11 spines were classified as grade 0, 1, and 2, respectively, of IVD degeneration (Figure 1A, top). Hematoxylin and eosin staining of the various IVDs showed much cell loss in grade 1 and 2 disks. As shown in Figure $1 \mathrm{~B}$, the average number of total cells per square millimeter were $447 \pm 117$ for the outer and $150 \pm 51$ for the inner AF of grade 0 specimens, $84 \pm 60$ for the AF of grade 1 specimens, and $3 \pm 3$ for the AF of grade 2 specimens. In grade 1 and 2 specimens there was no clear distinction between the inner and outer AF.

To determine the type of cell death observed in human IVDs, TUNEL assay was performed on grade 0 and 1 IVDs. Because very few cells could be detected, this experiment was not performed on grade 2 IVDs (Figure 1B). In all specimens examined, a significant number of AF cells showed positive TUNEL staining (Figure 2A), which indicates that apoptosis occurs during the degeneration process. Positive TUNEL staining for AF cells was $10 \pm 5 \%, 15 \pm 8 \%$, and $5 \pm 2 \%$ for the outer $A F$ of grade 0 IVDs, the inner AF of grade 0 IVDs, and the AF of grade 1 IVDs, respectively. To investigate the apoptotic pathway implicated in the IVD cell apoptosis, we performed immunostaining using anti-cytochrome $c$ and anti-Fas-L, which represents the initiation of MDA or death-receptordependent apoptosome. To localize the cytochrome $c$, we used an antibody that cannot penetrate the mitochon- 
A

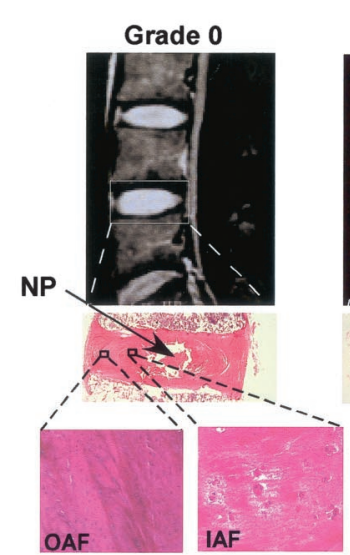

Grade 1

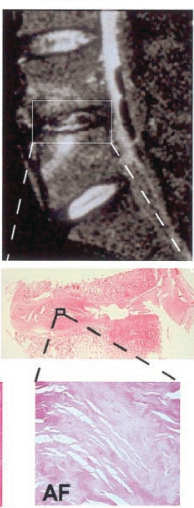

Grade 2
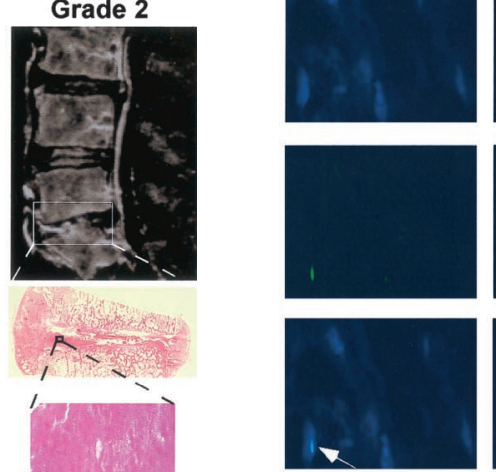

$B$
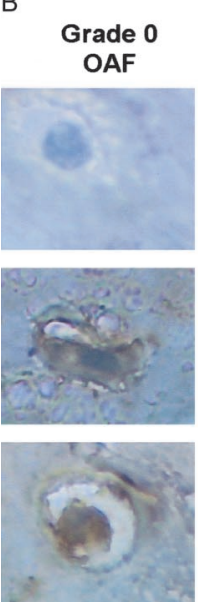

Grade 0 IAF
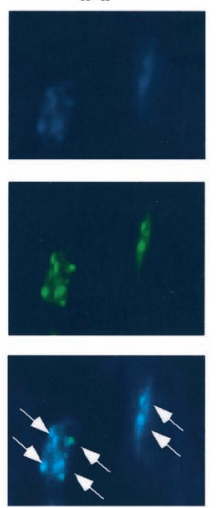

Grade 0 IAF
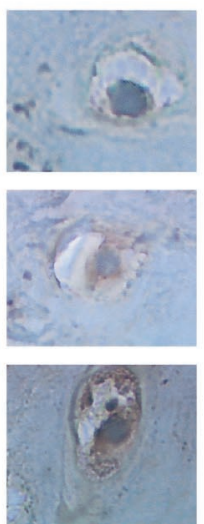

Grade 1

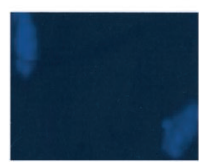

DAPI
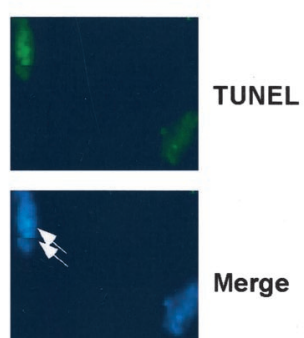

Grade 1
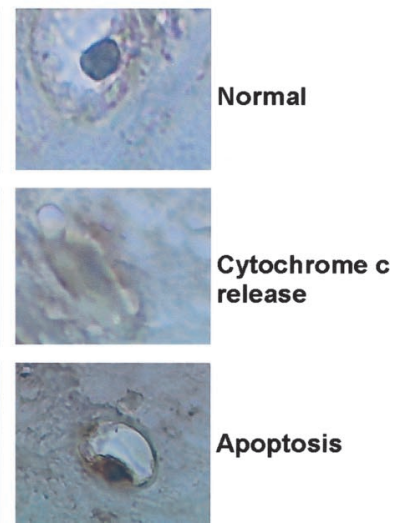

Figure 1. Cell death in human IVD degeneration. A: The top panels are representative MRIs of human lumbar spines. The selected areas represent the IVD categorized as grade 0 (no degeneration), grade 1 (moderate degeneration), and grade 2 (severe degeneration). The center panels revea the hematoxylin and eosin staining of midsagittal sections of the selected IVD. NP denotes nucleus pulposus. Bottom panels are representative views of the outer annulus fibrosus (OAF), inner annulus fibrosus (IAF) of grade 0 IVDs, and whole AF of grades 1 and 2 IVDs. Magnification: $\times 100$. B: Average number of cells per square millimeter from three views of each IVD at $\times 100$ magnification. ${ }^{*}$ indicates $P<0.05$ relative to OAF. $n$, number of IVDs examined.

drial membrane. Thus, this antibody only detects cytochrome $c$ released from the mitochondria. Most of the cell populations ( $92 \pm 5 \%$ for the outer AF of grade 0 IVDs, $88 \pm 7 \%$ for the inner AF of grade 0 IVDs, and $95 \pm 3 \%$ for the AF of grade 1 IVDs) showed normal nuclear morphological features, with little cytochrome $c$ immunostaining, which suggests the absence of cytochrome $c$ release from mitochondria in the cytosol of these cells (Figure 2B, top). A diffusive pattern of cytochrome $c$ immunoreactivity was found in the cytoplasm of some of the AF cells in both grade 0 and 1 specimens $(2 \pm 4 \%$ in the outer AF of grade 0 IVDs, $2 \pm 3 \%$ in the inner AF of grade 0 IVDs, and $2 \pm 2 \%$ in the AF of grade 1 IVDs, respectively), although these cells showed normal nuclear morphological features (Figure 2B, center). These results suggest the release of cytochrome $c$ from mitochondria to the cytosol in these cells, which is the key initiation event of mitochondria-dependent apoptosis. ${ }^{29}$ The cytochrome $c$ immunoreactivity was more robust in some cells in which typical apoptotic aspects, including cytoplasmic condensation and nuclear fragmentation, were detected $(9 \pm 4 \%$ for

Figure 2. Activation of the MDA in human IVD degeneration. A: DAPI (top) and TUNEL (center) staining of OAF, IAF of grade 0 IVDs, and AF of grade 1 disks. Bottom panels represent the merged images of DAPI and TUNEL staining. Arrows indicate the condensed and fragmented nuclei of apoptotic cells. Data are representative views from various IVDs. Magnification: $\times 400$. B: The corresponding cytochrome $c$ immunostaining of specimens shown in A. Top panels are normal AF cells (little immunostaining and normal nuclear morphological features). Center panels show cells having a diffusive pattern of immunoreactivity in the cytoplasm with normal nuclear morphological features, indicating the release of cytochrome $c$. Bottom panels represent cells with robust cytochrome $c$ staining, cytoplasmic condensation, and nuclear fragmentation. Magnification: $\times 1250$.

the outer AF of grade 0 IVDs, $12 \pm 6 \%$ for the inner AF of grade 0 IVDs, and $4 \pm 3 \%$ for the AF of grade 1 IVDs, respectively; Figure $2 \mathrm{~B}$, bottom). In contrast, very little Fas- $L$ immunoreactivity was detected in all AF cells regardless of the grade (data not shown). Taken together, these results suggest the involvement of apoptosis in cell death observed in the human IVD degeneration process, which is mediated through the activation of the MDA.

\section{Mechanical Stress Induces the MDA in IVD Degeneration in the Mouse Model}

Given the similar trend between human IVD degeneration, apoptosis, and the MDA, we used a mouse model to explore the role of mechanical stress in IVD degeneration. Mouse tail disks were subjected to an external compression device imposing a compressive stress in vivo 

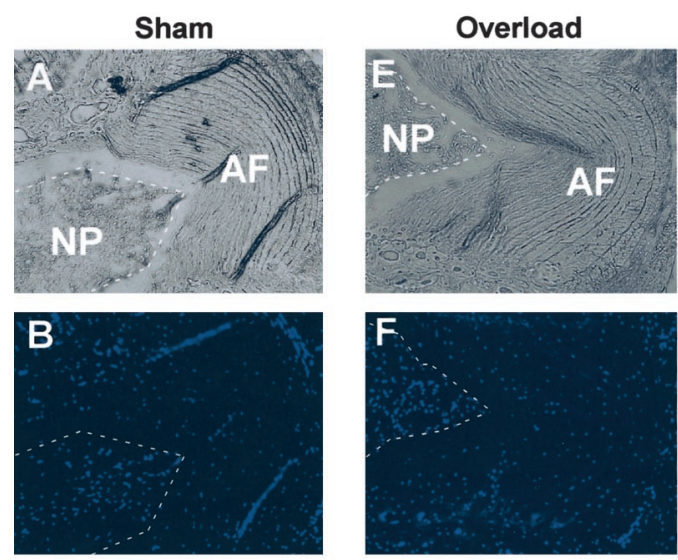

\section{DAPI}
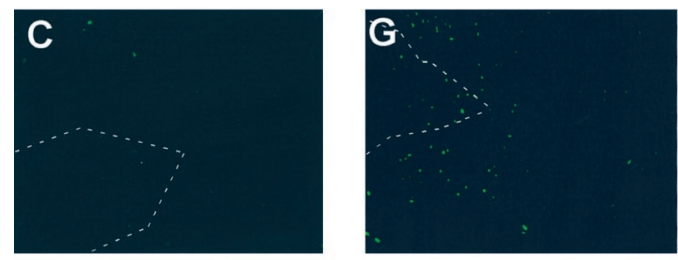

TUNEL
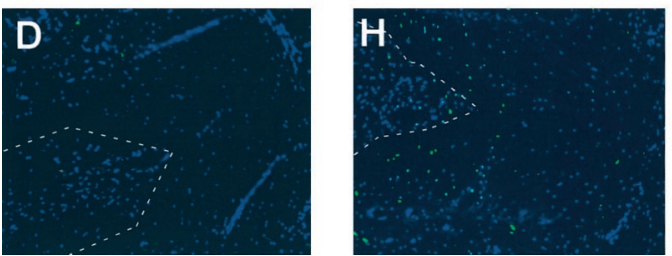

Merge

Figure 3. Apoptosis in mouse model of IVD degeneration induced by mechanical stress. Left panels are representative IVDs from mice receiving sham surgery; right panels are representative IVDs from mice receiving overload at $1.3 \mathrm{MPa}$ by an external compression device for 24 hours. $\mathbf{A}$ and $\mathbf{E}$ : Microscopic views of the AF and NP of IVDs. B and F: The corresponding DAPI staining. $\mathbf{C}$ and $\mathbf{G}$ : TUNEL staining on the same section. $\mathbf{D}$ and $\mathbf{H}$ : Merged images of DAPI and TUNEL staining. Magnification: $\times 100$. Note: the apoptotic AF cells are mainly located in the inner AF.
(1.3 MPa) between the ninth and $10^{\text {th }}$ caudal vertebrae for 24 hours. ${ }^{12}$ TUNEL staining showed a significant increase in apoptotic cells within the inner AF in animals subjected to the compressive stress (Figure 3). In contrast, few, if any, apoptotic cells were found in the outer AF of the animals and in sham controls receiving no compressive stress (Figure 3). To investigate the apoptotic pathway leading to the mechanical load-induced apoptosis observed in the mouse IVD, we performed immunostaining with anti-cytochrome $c$ or anti-Fas-L. In animals subjected to compressive stress, antigens recognized by anti-cytochrome $c$ were present in the cytosol of inner AF cells (Figure 4), which was similar to the anti-cytochrome $c$ staining results observed in human samples (Figure 2). Little or no immunoreactivity of anticytochrome $c$ was found in the cytosol of outer AF cells from animals receiving compressive stress or in AF cells from sham controls. In experiments using anti-Fas-L, immunoreactivity was found only in NP cells but not in AF cells, regardless of the loading (data not shown). Cells showing both TUNEL and cytochrome $c$ staining were mainly located in the inner AF. Furthermore, the inner AF cells showing positive TUNEL staining were fewer than those showing positive cytochrome $c$ staining. These qualitative results suggest that the activation of the MDA is the initial event leading to AF cell apoptosis and mechanical stress is the key regulator of this activation.

\section{Cyclic Stretch Induces IVD Cell Apoptosis in Vitro}

By taking advantage of the well-controlled mechanical and culture conditions in the in vitro experiments, we further deciphered the molecular mechanism by which mechanical stress induces IVD cell apoptosis. Rabbit AF cells cultured on collagen-coated membranes were kept

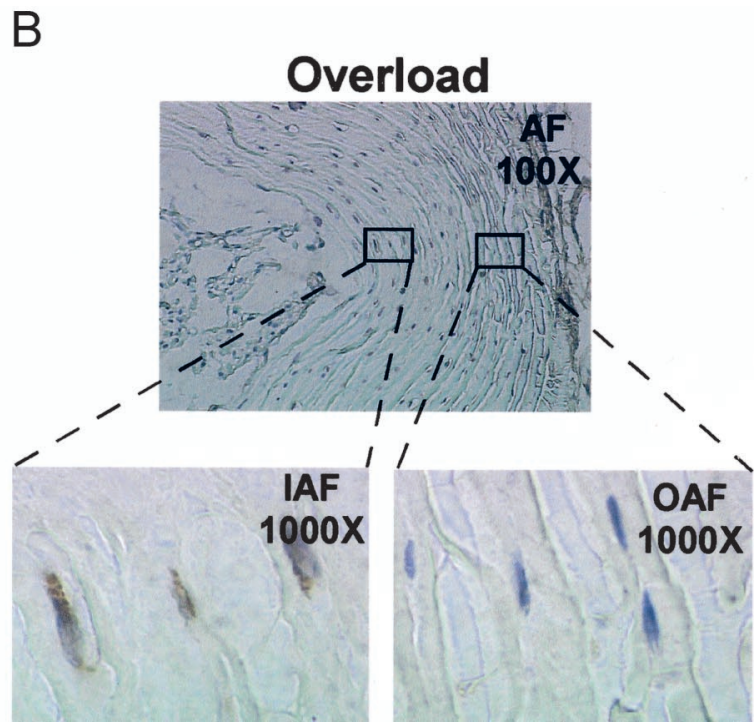

Figure 4. Activation of the MDA in mouse IVD degeneration induced by mechanical stress. A: A representative IVD from mice receiving sham surgery, whereas B represents an IVD receiving overload with the same mechanical conditions as those in Figure 3. Data shown are cytochrome $c$ immunostaining under different microscopic magnifications: top, $\times 100$; bottom, $\times 1000$. Note: high levels of cytochrome $c$ immunoreactivity is mainly found in the cytosol of inner AF cells of the IVD subjected to mechanical overload. 

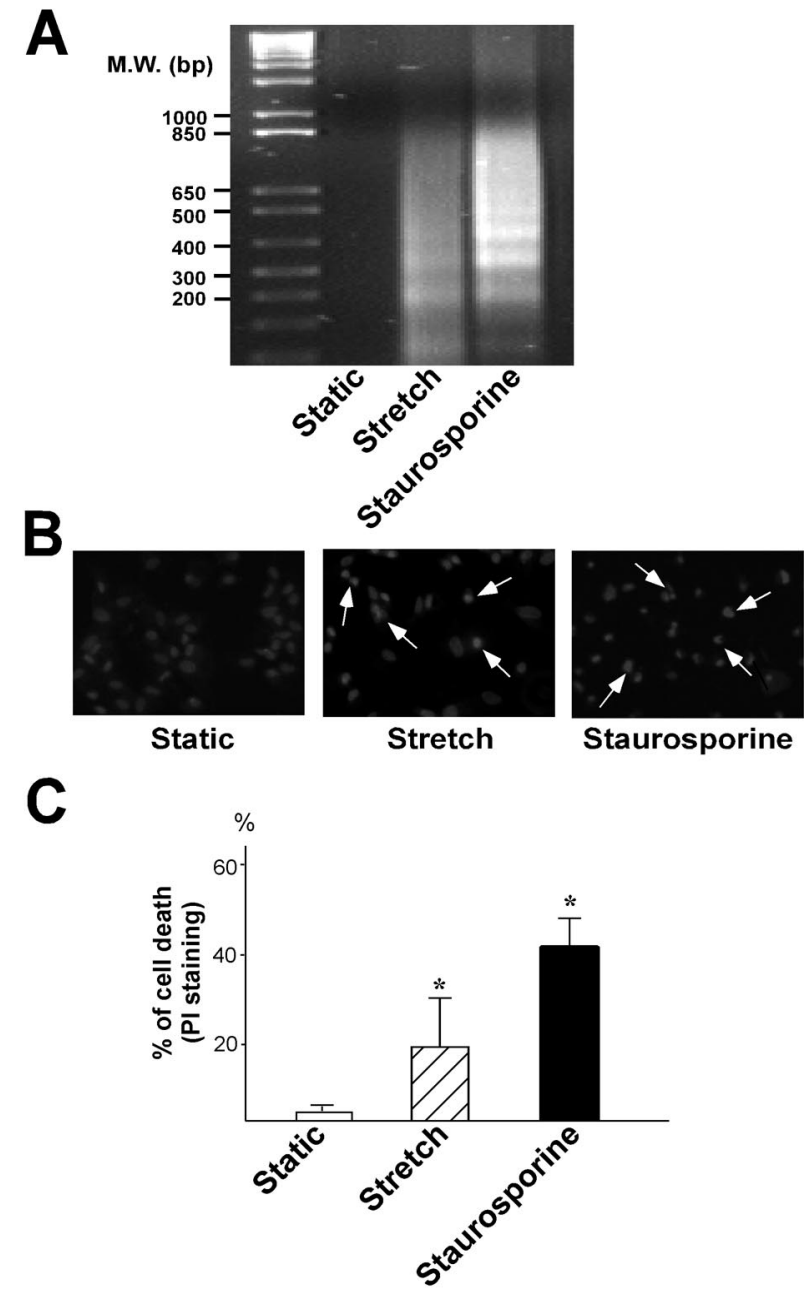

Figure 5. Cyclic stretch induces IVD cell apoptosis in vitro. Rabbit AF cells were kept as static controls, subjected to stretch at $15 \%$ for 24 hours, or treated with $1 \mu \mathrm{mol} / \mathrm{L}$ staurosporine for 10 hours. A: Genomic DNAs isolated from static, stretched, or staurosporine-treated AF cells were subjected to LM-PCR analysis followed by agarose gel electrophoresis. The data represent results of three independent experiments. B: The nuclear chromatin of $\mathrm{AF}$ cells was detected by Hoechst 33258 staining and indicates the cells undergoing apoptosis. Arrows indicate condensed and brightly stained nuclei. A representative section is shown at $\times 200$ magnification. C: Quantitative evaluation of apoptosis was assayed by FACS after PI staining. Results are presented as the percentage of cells below the $G_{0} / G_{1}$ peak (containing hypodiploid DNA) and are expressed as mean \pm SD of three independent experiments. * indicates $P<0.05$ relative to static controls.

as static controls or subjected to $15 \%$ cyclic stretch $(0.1$ $\mathrm{Hz}$ ) for 24 hours followed by LM-PCR, Hoechst staining, and flow cytometric analyses to detect apoptosis. A total of $15 \%$ area change represents the physiological limits of strain observed in IVDs. ${ }^{38}$ The mechanical stress imposed by the applied cyclic stretch caused AF cell apoptosis, as demonstrated by the DNA fragmentation (Figure 5A). In contrast, apoptosis was not detected in the static controls. DNA fragmentation was also seen in AF cells treated with $1 \mu \mathrm{mol} / \mathrm{L}$ staurosporine, a strong inducer of apoptosis. ${ }^{39}$ Cyclic stretch-induced AF cell apoptosis was further confirmed by Hoechst nuclear staining. As shown in Figure 5B, apoptosis-associated nuclear condensation and fragmentation increased in AF cells subjected to cyclic stretch or treated with staurosporine.
PI staining with FACS analysis was used to quantify the mechanical stress-induced apoptosis. As shown in Figure $5 \mathrm{C}$, cyclic stretch induced a significant increase in apoptosis $(17 \pm 11 \%)$, as compared with that in static controls $(2 \pm 1 \%)$. In the positive control experiments, $41 \pm 7 \%$ of the cells underwent apoptosis in response to staurosporine.

\section{Caspase-9 Regulates the Cyclic Stretch- Induced Apoptosis}

Since apoptosis in human and mouse IVDs is associated with the MDA (Figures 2 and 4), we investigated whether caspase-9, a cytochrome $c$ effector, is activated by cyclic stretch in vitro. Rabbit AF cells were subjected to cyclic stretch at $15 \%$, treated with staurosporine, or kept as static controls. In a separate set of experiments, cells were also exposed to Z-LEHD-FMK peptide, an irreversible caspase-9 inhibitor, before and during stretch experiments. After the various experiments, cells were lysed for caspase-9 activity assays, PI staining, DNA fragmentation, and $\Delta \Psi$ measurement. As shown in Figure $6 \mathrm{~A}$, compared to that under static conditions, caspase- 9 activity in the stretch- and staurosporine-treated cells was increased by $2.0 \pm 0$ and $2.7 \pm 0.1$ times, respectively. The release of cytochrome $c$ from mitochondria to the cytosol is necessary for caspase- 9 activation. ${ }^{29}$ Moreover, the alteration of $\Delta \Psi$ leads to the release of cytochrome $c$, thus decreasing the mitochondrial uptake of $\mathrm{DiOC}_{6}{ }^{40}$ Therefore, we investigated the changes in $\Delta \Psi$ in response to cyclic stretch by FACS measurement of the $\mathrm{DiOC}_{6}$ fluorescence. As shown in Figure 6B, mechanical stress caused by cyclic stretch and staurosporine (positive control) decreased the $\Delta \Psi$, as indicated by the increased proportion of cells with low $\mathrm{DiOC}_{6}$ fluorescence compared with the unaffected $\Delta \Psi$ in static controls (Figure 6B). PI staining and DNA fragmentation analyses further revealed that the stretch-induced AF cell apoptosis was much attenuated by the caspase-9 inhibitor (Figure $6, C$ and $D)$.

The activation of the MDA can be initiated indirectly by up-regulated caspase-8. ${ }^{30}$ To investigate whether the death receptor-caspase-8 apoptotic pathway is also involved in mechanical stress-induced AF cell apoptosis, we analyzed the caspase- 8 activity in response to cyclic stretch. We observed that the caspase-8 activity in AF cells was not affected by the imposed cyclic stretch. Furthermore, the inclusion of an inhibitor specific to caspase-8 (ie, $100 \mu \mathrm{mol} / \mathrm{L}$ Z-IETD-FMK) had no effect on the stretch-induced apoptosis assessed by PI staining and DNA fragmentation assays (data not shown). These results and data presented in Figure 6 suggest that mechanical stress activates the MDA in AF cells, which depend on caspase-9 but not caspase-8.

\section{Discussion}

IVD degeneration is found in more than $40 \%$ of patients who experience low back pain. ${ }^{8}$ Although ample evi- 
A

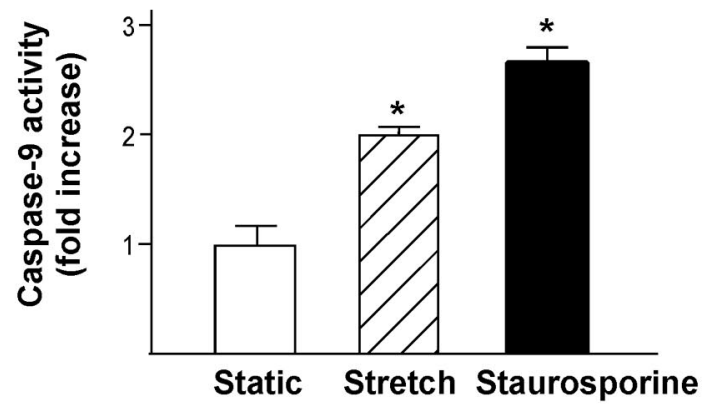

B

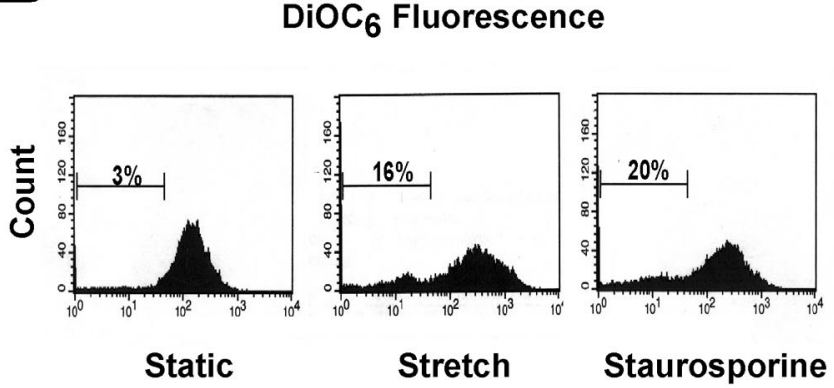

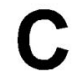

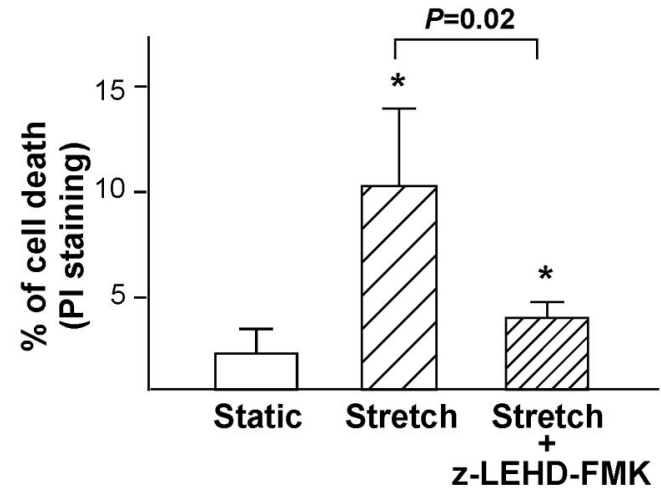

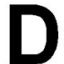

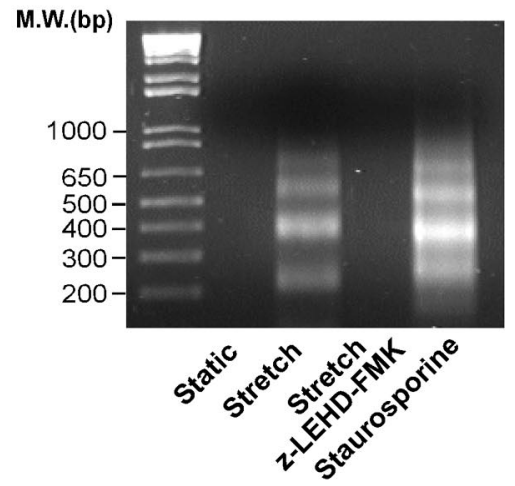

Figure 6. Cyclic stretch-induced apoptosis is initiated by MDA. Rabbit AF cells were kept as static controls (A and B), stretched at $15 \%$ for 24 hours, or treated with $1 \mu \mathrm{mol} / \mathrm{L}$ staurosporine or kept as static controls (C and D), stretched at 15\% for 24 hours, or treated with 100 $\mu \mathrm{mol} / \mathrm{L}$ Z-LEHD-FMK. A: The caspase-9 activity in cell lysates was measured by detecting the cleavage of LEHD- $p$-NA, determined by use of a colorimetric plate reader at $405 \mathrm{~nm}$. Results are expressed as mean \pm SD from three independent experiments. B: $\Delta \Psi$ was assayed by FACS after DiOC 6 staining. The percentage represents the fraction of cells with decreased DiOC 6 fluorescence. C: Quantitative evaluation of apoptosis was assayed by FACS after PI staining. Results are presented as the percentage of cells below the $G_{0} / G_{1}$ peak (containing hypodiploid DNA) and expressed as mean \pm SD from three independent experiments. D: DNA fragmentation was examined after LM-PCR analysis of genomic DNA followed by agarose gel electrophoresis. * indicates $P<0.05$ relative to static controls.

dence from clinical studies links physical exertion to low back pain and IVD degeneration, the involved molecular mechanism is still unclear, ${ }^{13}$ mainly because of the difficulty in obtaining intact IVD tissue from patients for pathophysiological examination. However, a mouse model of IVD degeneration has been developed recently. ${ }^{12}$ In this study, we used human samples, the mouse model, and in vitro mechanical loading on cultured rabbit IVD cells to investigate the mechanical basis of IVD degeneration, which can lead to low back pain. We observed that apoptosis is a major event associated with IVD degeneration. Induced by mechanical overload, this pathophysiological process is mediated through the activation of the MDA, including cytochrome $c$ release and caspase- 9 activation.

MRI and histological analysis on whole IVDs from cadavers of patients revealed that tissue degeneration is associated with cell death in IVDs (Figure 1). This result is consistent with a previous study involving IVD explants. ${ }^{21}$ To our knowledge, few histological studies on whole IVDs from patients with low back pain has been performed previously, mainly because of the lack of invasive procedures to dissect and remove impaired IVDs. Also, it is difficult to differentiate IVD tissue from surrounding tis- sues such as bone or vertebral end plates and AF from NP for biochemical analyses. The T2 signal intensity of $\mathrm{MRI}$ is a useful clinical tool to reveal the hydration status of the IVD that corresponds well with the degree of degeneration. ${ }^{41}$ Associating the degeneration of human IVDs, assessed by MRI, with histological results of specimens collected from the same IVD, we observed fewer cells in moderate and highly degenerated IVDs (grade 1 and 2) than in normal disks (grade 0; Figure 1, A and B). This cell loss can be attributed in part to AF cell apoptosis, because few AF cells with positive TUNEL staining were detected in IVDs (Figure 2A). Because the whole intact IVDs were taken from spines of deceased patients, it is likely that the observed apoptotic process was due in part to the time lag (48 hours) between the death of the patient and the dissection of the spine from the cadaver. The development of the anterior surgical approach could resolve this technical limitation. It is also noted that the TUNEL preferentially labels apoptotic cells but can also label DNA fragments generated during the late period in necrosis. Thus, we cannot rule out that some TUNELpositive cells might have been necrotic cells.

By using an antibody that detects only the cytosolic cytochrome $c,{ }^{37}$ we found the release of cytochrome $c$ in 
the cytoplasm of AF cells in both grade 0 and 1 specimens (Figure 2B, center). These cells, having normal nuclear morphological features, revealed that the MDA would initiate AF cell apoptosis. Moreover, AF cells showing cytochrome $c$ immunoreactivity with condensed cytoplasm and fragmented nuclei suggest that some cells experienced a later stage of apoptosis (Figure 2B, bottom). In contrast, little if any, Fas-L staining was observed in AF cells in these specimens. The data suggest that the MDA but not Fas-L-related signaling is a major apoptotic pathway implicated in the degeneration of human IVDs. Apoptosis and cytochrome $c$ release were observed in IVDs with normal T2 MRI signal intensity (grade 0), which clinicians consider to be a healthy state. This may demonstrate an early but active degeneration process in morphologically normal tissues: clearly, apoptosis initiates in normal disks and must precede the morphological alterations that are pathognomonic of degenerated disks. While we do not know the premortem loading history for these human tissues, it may be that mechanical stress accelerates the physiological process of aging, leading to an acute degeneration, which is a pathological state of IVD degeneration.

A mouse model further supports the acute IVD degeneration caused by the MDA in AF cells in response to mechanical overload. This experimental system with accelerated IVD degeneration has its advantages in that 1) the newborn mouse disk has a structural organization and cell population similar to that in young people, ${ }^{42,43} 2$ ) the mouse IVD degeneration mimics that in adults, ${ }^{44,45}$ and 3 ) the applied mechanical overload results in a degeneration phenomenon similar to that observed in overloaded human IVDs. ${ }^{12,14,46}$ Coinciding with results obtained from human specimens with IVD degeneration (Figures 1 and 2), mechanical overload on mouse spines increased the number of apoptotic AF cells and the release of cytochrome $c$ (Figures 3 and 4). Furthermore, the absence of positive Fas- $L$ staining in AF cells indicates that the Fas signaling is not the major pathway mediating the mechanical stress-induced AF cell apoptosis. These results suggest that mechanical overload can be a key stress, leading to excessive apoptosis of AF cells, which is mediated through the MDA. The presence of anti-Fas immunoreactivity in the NP but not in AF cells of mouse IVD is currently unknown. One possible explanation is that the distinct embryological origin of NP and AF cells may lead to higher apoptosis in NP cells even under physiological conditions. To reach the homeostasis, NP might be programmed to death by the activation of death receptors, regardless of the loading conditions.

Results from experiments involving human samples and mouse models are supported by experiments showing that mechanical stress-induced rabbit AF cell apoptosis occurs via a capsase-9-dependent pathway (Figures 5 and 6). Activated in cells secondary to various stimuli, including UV irradiation, hypoosmolarity, growth factor deprivation, DNA-damage oxidative stress, heat shock, cytotoxic drugs, ischemia, and ceramide, the MDA is a major mediator in maintaining cellular homeostasis. ${ }^{47-50}$ The role of the MDA in IVD degeneration is reminiscent of the process of other degenerative dis- orders such as amyotrophic lateral sclerosis (ALS), Alzheimer's disease, and Parkinson's disease. Cytochrome $c$ release and caspase- 9 activation occur in the cells of the spinal cord during the neurodegenerative process of ALS. ${ }^{51}$ The Bid-Bax pathway, lacking caspase-8 activation, has been suggested to activate the MDA involved in ALS. ${ }^{51,52}$ In Alzheimer's and Parkinson's diseases, the upstream molecules regulating the MDA involve p53 and reactive oxygen species (ROS). ${ }^{53,54}$ The lack of Fas-L and caspase-8 activation in our in vivo and in vitro results, suggesting that mechanical overload activates death receptor(s), which in turn up-regulate(s) the MDA, is less likely. However, the involvement of the death receptor apoptosome in the later stage of the degeneration process or a caspase-8-independent Bid activation as observed in ALS cannot be completely ruled out. Another alternative is that ROS may activate the MDA in response to mechanical overload. Results from a recent study showed that ROS in cardiac myocytes were increased by mechanical stretch at high amplitudes, which led to apoptosis. ${ }^{55}$ Mechanical stretch has been shown to induce apoptosis in myocytes via the activation of the transcriptional factor p53, leading to a decrease of the $\mathrm{Bcl}-2$ :Bax ratio, which suggests the participation of the MDA. ${ }^{56,57}$ Furthermore, p38 MAPK activation of p53 has been shown to mediate apoptosis of vascular smooth muscle cells induced by mechanical stretch. ${ }^{58,59} \mathrm{~A}$ decreased $\mathrm{Bcl}-2$ :Bax ratio was found in these cells as well. ${ }^{59}$ Data from the current study, together with previous results, suggest that ROS production, p38 MAPK activation, p53dependent Bax induction, cytochrome $c$ release, and caspase- 9 activation could constitute the mechanotransduction pathway, leading to apoptosis in response to mechanical overload.

In conclusion, evidence from the current study suggests that the MDA could be associated with AF cell apoptosis in human IVD degeneration in response to mechanical overload. Understanding this mechanism may benefit the development of specific treatments for patients with low back pain.

\section{Acknowledgments}

We thank Drs. Maite Corvol, Michel Revel, and Serge Poiraudeau for their critical reviews, and Dr. Kitty Formentin, Barbara Walter, Dr. Takuya Fukazawa, and Andre Morgan for technical assistance.

\section{References}

1. Annunen S, Paassilta P, Lohiniva J, Perala M, Pihlajamaa T, Karppinen J, Tervonen O, Kroger H, Lahde S, Vanharanta H, Ryhanen L, Goring $\mathrm{HH}$, Ott J, Prockop DJ, Ala-Kokko L: An allele of COL9A2 associated with intervertebral disc disease. Science 1999, 285:409412

2. Kawaguchi Y, Osada R, Kanamori M, Ishihara H, Ohmori K, Matsui H, Kimura T: Association between an aggrecan gene polymorphism and lumbar disc degeneration. Spine 1999, 24:2456-2460

3. Hoogendoorn WE, van Poppel MNM, Bongers PM, Koes BW, Bouter LM: Systematic review of psychosocial factors at work and private life as risk factors for back pain. Spine 2000, 25:2114-2125 
4. Paassilta P, Lohiniva J, Goring HH, Perala M, Raina SS, Karppinen J, Hakala M, Palm T, Kroger H, Kaitila I, Vanharanta H, Ott J, Ala-Kokko $\mathrm{L}$ : Identification of a novel common genetic risk factor for lumbar disk disease. JAMA 2001, 285:1843-1849

5. Pincus T, Burton AK, Vogel S, Field AP: A systematic review of psychological factors as predictors of chronicity/disability in prospective cohorts of low back pain. Spine 2002, 27:109-120

6. Kang JD, Stefanovic-Racic M, McIntyre LA, Georgescu HI, Evans CH: Toward a biochemical understanding of human intervertebral disc degeneration and herniation. Spine 1997, 22:1065-1073

7. Luoma K, Riihimaki H, Luukkonen R, Raininko R, Viikari-Juntura E, Lamminen A: Low back pain in relation to lumbar disc degeneration. Spine 2000, 25:487-492

8. Freemont AJ, Watkins AJ, Watkins A, Le Maitre C, Jeziorska M, Hoyland JA: Current understanding of cellular and molecular events in intervertebral disc degeneration: implications for therapy. J Pathol 2002, 196:374-379

9. Fairbank J: Clinical importance of the intervertebral disc, or back pain for biochemists. Biochem Soc Trans 2002, 30:829-831

10. Urban JP, Robert S: Degeneration of the intervertebral disc. Arthritis Res 2003, 5:120-130

11. Videman T, Nurminen M, Troup JDG: Lumbar spinal pathology in cadaveric material in relation to history of back pain, occupation, and physical loading. Spine 1990, 15:728-740

12. Lotz JC, Colliou OK, Chin JR, Duncan NA, Liebenberg E: Compression-induced degeneration of the intervertebral disc: an in vivo mouse model and finite-element study. Spine 1998, 23:2493-2506

13. Adams MA, Dolan $P$ : Could sudden increases in physical activity cause degeneration of intervertebral discs? Lancet 1997, 350:734735

14. Lotz JC, Chin JR: Intervertebral disc cell death is dependent on the magnitude and duration of spinal loading. Spine 2000, 25:1477-1483

15. Kang JD, Goergescu HI, Mclntyre-Larkin L, Stefanovic-Racic M, Donaldson W, Evans $\mathrm{CH}$ : Herniated lumbar intervertebral discs spontaneously produce matrix metalloproteinases, nitric oxide, interleukin-6, and prostaglandin $\mathrm{E}_{2}$. Spine 1996, 21:271-277

16. Antoniou J, Steffen T, Nelson F, Winterbottom N, Hollander AP, Poole RA, Aebi M, Alini M: The human lumbar intervertebral disc. J Clin Invest 1996, 98:996-1003

17. Poiraudeau S, Monteiro I, Anract P, Blanchard O, Revel M, Corvol MT: Phenotypic characteristics of rabbit intervertebral disc cells: comparison with cartilage cells from the same animals. Spine 1999, 24:837844

18. Rannou F, Corvol MT, Hudry C, Anract P, Blanchard O, Tsagris L, Revel M, Poiraudeau S: Sensitivity of anulus fibrosus cells to interleukin $1 \beta$ : comparison with articular chondrocytes. Spine 2000, 25: $17-23$

19. Haro H, Crawford HC, Fingleton B, Shinomiya K, Spengler DM, Matrisian LM: Matrix metalloproteinase-3-dependent generation of a macrophage chemoattractant in a model of herniated disc resorption. J Clin Invest 2000, 105:133-141

20. Buckwalter JA: Aging and degeneration of the human intervertebral disc. Spine 1995, 20:1307-1314

21. Gruber HE, Hanley EN: Analysis of aging and degeneration of the human intervertebral disc. Spine 1998, 23:751-757

22. Ishihara H, McNally DS, Urban JPG, Hall AC: Effects of hydrostatic pressure on matrix synthesis in different regions of the intervertebral disk. J Appl Physiol 1996, 80:839-846

23. Handa $T$, Ishihara H, Ohshima H, Osada R, Tsuji H, Obata K: Effects of hydrostatic pressure on matrix synthesis and matrix metalloproteinase production in the human lumbar intervertebral disc. Spine 1997, 22:1085-1091

24. Hutton WC, Toribatake Y, Elmer WA, Ganey TM, Tomita K, Whitesides TE: The effect of compressive force applied to the intervertebral disc in vivo. Spine 1998, 23:2524-2537

25. Hutton WC, Elmer WA, Boden SD, Hyon S, Toribatake Y, Tomita K, Hair GA: The effect of hydrostatic pressure on intervertebral disc metabolism. Spine 1999, 24:1507-1515

26. Horvitz HR: Genetic control of programmed cell death in the nematode Caenorhabditis elegans. Cancer Res 1999, 59:1701-1706

27. Hengartner MO: The biochemistry of apoptosis. Nature 2000, 407 770-776

28. Nicholson DW: From bench to clinic with apoptosis-based therapeutic agents. Nature 2000, 407:810-816
29. Kroemer G, Reed JC: Mitochondrial control of cell death. Nat Med 2000, 6:513-519

30. Li H, Zhu H, Xu CJ, Yuan J: Cleavage of BID by caspase 8 mediates the mitochondrial damage in the Fas pathway of apoptosis. Cell 1998, 94:491-501

31. Karppinen J, Paakko E, Raina S, Tervonen O, Kurunlahti M, Nieminen P, Ala-Kokko L, Malmivaara A, Vanharanta $\mathrm{H}$ : Magnetic imaging resonance findings in relation to the COL9A2 tryptophan allele among patients with sciatica. Spine 2002, 27:78-83

32. Lee TS, Chau LY: Fas/Fas ligand-mediated death pathway is involved in oxLDL-induced apoptosis in vascular smooth muscle cells. Am J Physiol Cell Physiol 2001, 280:C709-C718

33. Rannou F, Poiraudeau S, Foltz V, Boiteux M, Corvol M, Revel M: Monolayer anulus fibrosus cell cultures in a mechanically active environment: local culture condition adaptations and cell phenotype study. J Lab Clin Med 2000, 136:412-421

34. Sotoudeh M, Jalali S, Usami S, Shyy JY, Chien S: A strain device imposing dynamic and uniform equi-biaxial strain to cultured cells. Ann Biomed Eng 1998, 26:181-189

35. Wang J, Zhen L, Klug MG, Wood D, Wu X, Mizrahi J: Involvement of caspase 3- and 8-like proteases in ceramide-induced apoptosis of cardiomyocytes. J Card Fail 2000, 6:243-249

36. Sumitomo M, Ohba M, Asakuma J, Asano T, Kuroki T, Asano T, Hayakawa M: Protein kinase $C \delta$ amplifies ceramide formation via mitochondrial signaling in prostate cancer cells. J Clin Invest 2002, 109:827-836

37. Matz PG, Fujimura M, Lewen A, Morita-Fujimura Y, Chan PH: Increased cytochrome c-mediated DNA fragmentation and cell death in manganese-superoxide dismutase-deficient mice after exposure to subarachnoid hemolysate. Stroke 2001, 32:506-515

38. Broberg KB: On the mechanical behaviour of intervertebral discs. Spine 1983, 8:151-165

39. Bertrand R, Solary E, O'Connor P, Kohn KW, Pommier Y: Induction of a common pathway of apoptosis by staurosporine. Exp Cell Res 1994, 211:314-321

40. Li PF, Dietz R, von Harsdorf R: p53 regulates mitochondrial membrane potential through reactive oxygen species and induces cytochrome c-independent apoptosis blocked by Bcl-2. EMBO J 1999, 18:6027-6036

41. Stadnik TW, Lee RR, Coen HL, Neirynck EC, Buisseret TS, Osteaux MJ: Annular tears and disk herniation: prevalence and contrast enhancement on MR images in the absence of low back pain or sciatica. Radiology 1998, 206:49-55

42. Hickey DS, Hukins DWL: Aging changes in the macromolecular organization of the intervertebral disc. Spine 1982, 7:234-242

43. Higuchi M, Abe K, Kaneda K: Changes in the nucleus pulposus of the intervertebral disc in bipedal mice. Clin Orthop 1983, 175:251-257

44. Higuchi M, Abe K: Postmortem changes in ultrastructures of the mouse intervertebral disc. Spine 1987, 12:48-52

45. Miyamoto S, Yonenobu K, Ono K: Experimental cervical spondylosis in the mouse. Spine 1991, 16:S495-S500

46. Pritzker KPH: Animal models for osteoarthritis: processes, problems and prospects. Ann Rheum Dis 1994, 53:406-420

47. Rich $\mathrm{T}$, Allen RL, Wyllie AH: Defying death after DNA damage. Nature 2000, 407:777-783

48. Adrain C, Creagh EM, Martin SJ: Apoptosis-associated release of Smac/DIABLO from mitochondria requires active caspases and is blocked by Bcl-2. EMBO J 2001, 20:6627-6636

49. Schuler M, Green DR: Mechanisms of p53-dependent apoptosis. Biochem Soc Trans 2001, 29:684-688

50. Movsesyan VA, Yakovlev AG, Dabaghyan EA, Stoica BA, Faden AI: 2002 Ceramide induces neuronal apoptosis through the caspase-9/ caspase-3 pathway. Biochem Biophys Res Commun 2002, 299:201207

51. Guegan C, Vila M, Rosoklija G, Hays AP, Przedborski S: Recruitment of the mitochondrial-dependent apoptotic pathway in amyotrophic lateral sclerosis. J Neurosci 2001, 21:6569-6576

52. Guegan C, Vila M, Teissman P, Chen C, Onteniente B, Li M, Friedlander RM, Przedborski S: Instrumental activation of bid by caspase- 1 in a transgenic mouse model of ALS. Mol Cell Neurosci 2002, 20:553-562

53. Tanaka S, Takehashi M, Matoh N, lida S, Suzuki T, Futaki S, Hamada $H$, Masliah E, Sugiura Y, Ueda K: Generation of reactive oxygen 
species and activation of NF- $\kappa$ B by non-A $\beta$ component of Alzheimer's disease amyloid. J Neurochem 2002, 82:305-315

54. Tatton WG, Chalmers-Redman R, Brown D, Tatton N: Apoptosis in Parkinson's disease: signals for neuronal degradation. Ann Neurol 2003, 53:S61-72

55. Pimentel DR, Amin JK, Xiao L, Miller T, Viereck J, Oliver-Krasinski J, Baliga R, Wang J, Siwik DA, Singh K, Pagano P, Colucci WS, Sawyer DB: Reactive oxygen species mediate amplitude-dependent hypertrophic and apoptotic responses to mechanical stretch in cardiac myocytes. Circ Res 2001, 89:453-460

56. Leri A, Claudio PP, Li Q, Wang X, Reiss K, Wang S, Malhotra A, Kajstura J, Anversa P: Stretch-mediated release of angiotensin II induces myocyte apoptosis by activating p53 that enhances the local renin-angiotensin system and decreases the Bcl-2-to-Bax protein ratio in the cell. J Clin Invest 1998, 101:1326-1342

57. Leri A, Fiordaliso F, Setoguchi M, Limana F, Bishopric NH, Kajstura J, Webster K, Anversa P: Inhibition of p53 function prevents reninangiotensin system activation and stretch-mediated myocyte apoptosis. Am J Pathol 2000, 157:843-857

58. Mayr M, Li C, Zou Y, Huemer U, Hu Y, Xu Q: Biomechanical stressinduced apoptosis in vein grafts involves p38 mitogen-activated protein kinases. EMBO J 2000, 14:261-270

59. Mayr M, Hu Y, Hainaut $H, X u$ Q: Mechanical stress-induced DNA damage and rac-p38MAPK signal pathways mediate p53-dependent apoptosis in vascular smooth muscle cells. EMBO J 2002, 16:14231425 